\title{
Differential EFfects of Ballistic Versus Sensorimotor Training on Rate of Force Development aNd Neural ACtivation in Humans
}

\author{
Markus Gruber, ${ }^{1}$ Stefante B.H. Gruber, ${ }^{1}$ Wolfgang Taube, ${ }^{1}$ Martin Schubert, ${ }^{2}$ \\ SANDra C. BeCK, ${ }^{2}$ and Albert Gollhofer ${ }^{1}$ \\ 'Department of Sport Science, University of Freiburg, Freiburg, Germany; ${ }^{2}$ Department of Clinical Neurology and \\ Neurophysiology, University of Freiburg, Freiburg, Germany.
}

\begin{abstract}
Gruber, M., S.B.H. Gruber, W. Taube, M. Schubert, S.C. Beck, and A. Gollhofer. Differential effects of ballistic versus sensorimotor training on rate of force development and neural activation in humans. J. Strength Cond. Res. 21(1):274-282. 2007.-Balancing exercises on instable bases (sensorimotor training [SMT]) are often used in the rehabilitation process of an injured athlete to restore joint function. Recently it was shown that SMT was able to enhance rate of force development (RFD) in a maximal voluntary muscle contraction. The purpose of this study was to compare adaptations on strength capacity following ballistic strength training (BST) with those following an SMT during a training period of 1 microcycle (4 weeks). Maximum voluntary isometric strength (MVC), maximum RFD (RFDmax) and the corresponding neural activation of M. soleus (SOL), M. gastrocnemius (GAS), and M. tibialis anterior (TIB) were measured during plantar flexion in 33 healthy subjects. The subjects were randomly assigned to a SMT, BST, or control group. RFDmax increased significantly stronger following BST $(48 \pm 16 \% ; p<0.01)$ compared to SMT $(14 \pm 5 \% ; p<0.05)$, whereas MVC remained unchanged in both groups. Median frequencies of the electromyographic power spectrum during the first $200 \mathrm{~ms}$ of contraction for GAS increased following both BST $(45 \pm 21 \% ; p<0.05)$ and SMT $(45 \pm 22 \% ; p<0.05)$, but median frequencies for SOL increased only after SMT $(13 \pm 4 \% ; p<$ 0.05 ). Additionally, mean amplitude voltage increased following BST for SOL $(38 \pm 12 \% ; p<0.01)$ and for GAS $(73 \pm 23 \% ; p<$ 0.01 ) during the first $100 \mathrm{~ms}$, whereas it remained unchanged after SMT. It is concluded that BST and SMT may induce different neural adaptations that specifically affect recruitment and discharge rates of motor units at the beginning of voluntary contraction. Specific neural adaptations indicate that SMT might be used complementarily to BST, especially in sports that require contractile explosive properties in situations with high postural demands, e.g., during jumps in ball sports.
\end{abstract}

KEY WoRDs. explosive strength, neural drive, M-wave, plantar flexion

\section{INTRODUCTION}

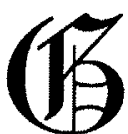
rowing interest has been given to the ability of the muscle to produce maximum power and high force values within short periods of time. Explosive muscle strength is considered to be of major importance in many sports that involve ballistic muscle contractions (30) and in injury-related situations, in which a joint has to be stiffened in a very short time period (14). More functionally, training in the elderly could reduce the risk of falls by fast activation of the mus. cles concomitant with improvements of postural control (23).

It has been shown that adaptations in muscular performance are highly specific depending on the training loads (22). Therefore, different training methods have been established with the aim of developing distinct strength qualities. Training with high loads (high-intensity strength training) improved maximum voluntary iso. metric strength (MVC) considerably with only minor changes in the rate of force development (RFD), whereas moderate loads that were accelerated with maximal effort (ballistic strength training [BST]) had minor effects on the MVC but enhanced RFD extensively $(10,18,29)$. The underlying neural mechanisms of a BST were recently examined by Van Cutsem et al. (36). Through analysis of single motor unit (MU) recordings, earlier recruitment of high-threshold MUs, enhanced maximal discharge rates of MUs, and an increased incidence of "doublets" during ballistic contractions after 3 months of dynamic training were demonstrated for $\mathrm{M}$. tibialis anterior (TIB). The authors suggested that these adaptations were caused by an enhanced neural drive to the motoneurons of the trained muscle. Most probably the actuating stimulus is the execution of a voluntary motor command with the intention to accelerate a load as fast as possible $(3,39)$.

Recently, comparable effects on RFD were reported following a sensorimotor training (SMT) (17). The authors reported an enhanced maximal RFD (RFDmax) of $33 \%$ for voluntary isometric leg press exercises after a 4-week training period. This is remarkable as during SMT the muscles are innervated constantly with only submaximal intensity in order to ensure balance. The authors therefore suggested that afferent contributions might play an important role for the observed adaptations in explosive strength. Recently, Grande and Cafarelli (16) examined the recruitment thresholds and the firing rates of single MUs when recruited via the Ia afferent pathway compared to voluntary activation during force matched isometric knee extensions. The authors reported that MUs of $M$. vastus lateralis were recruited earlier but with lower firing rates when activated via the Ia afferent pathway compared to a voluntary activation. This clearly indicates that recruitment thresholds and firing rates of MUs depend on the amount of afferent input.

If the muscle activation during SMT is differentially organized compared to BST, it could be hypothesized that neural adaptations should differ as well. This could be quite important because it is known that the increases in strength observed during the first weeks of a specific training (e.g., a micracycle) may primarily result from considerable neural adaptations (26). Therefore, the purpose of this study was to compare specific adaptations in explosive strength capacity and neural activation between BST and SMT for 1 microcycle. 


\section{METHODS}

\section{Experimental Approach to the Problem}

To test our hypothesis, specific adaptations for SMT and BST were compared in a controlled longitudinal training study. The training period lasted 4 weeks (microcycle) in order to ensure neuromuscular adaptations. Neural vs. muscular improvements were verified by an analysis of twitch torques that were released by supramaximal nerve stimulations. In both training regimens, focus was set on ankle plantar flexors. Plantar flexors, especially triceps strae muscles, are functionally important in those sport disciplines that involve jumping or running. Here, development of high forces within a short time period (e.g., muscle power) is of vital importance for performance.

Explosive contractions served as reference to elaborate strength and power adaptation in both training regimens. These tests were performed isometrically in order to ensure high reliability. From a functional point of view contractile impulses were evaluated in order to predict segmental angular velocity in nonisometric conditions. Analysis of torque and the corresponding electromyography (EMC) of agonistic and antagonistic muscles allowed detection of functional mechanisms of the observed ad aplations. The following sections will explain and specify the different parameters.

\section{Subjects}

Thirty-three subjects participated in the study. All subjects were healthy with no neurological disorders and no injuries of the lower extremities. They were not involved in any other systematic training during the experiment and had not engaged in BST or SMT before. All the subjects gave their written informed consent before participating in the study. They were randomly assigned to 1 of 3 groups: BST ( 6 male and 5 female subjects; age, $27 \pm$ 6 years; height, $1.72 \pm 0,08 \mathrm{~m}$; weight, $68 \pm 13 \mathrm{~kg}$ ), SMT (5 male and 6 female subjects; age, $25 \pm 3$ years; height, $1.72 \pm 0.08 \mathrm{~m}$, weight, $64 \pm 9 \mathrm{~kg}$ ), or control (CON: 6 male and 5 fernale subjects; age, 26 years; height, $1.73 \pm 0.07 \mathrm{~m} ;$ weight, $68 \pm 8 \mathrm{~kg}$ ). The study was approved by the local ethics committee and conducted according to the Declaration of Helsinki.

\section{Procedures}

Training. The SMT and BST groups trained over a period of 4 weeks, with a total of 16 training sessions. All sessions were documented, surveyed, and supervised by the authors of the study. The SMT and BST groups trtined according to protocols that have been used previously $(10$, $17,32,36$ ). Figure 1 gives a detailed description of the respective training regimens Care was taken to match training duration and intensity between the BST and SMT groups. The SMT was characterized by a complex activation pattern in the muscles encompassing the ankle joint in order to maintain or regain balance. The BST, in contrast, was characterized by isolated voluntary explosive activations of plantar and dorsal flexors in order to move a light load as fast as possible. For both training regimens, the procedure was controlled very carefully by the authors of this study to ensure maximum effort of the subjects. The training was intensified after 8 sessions by increasing the number of sets to 6 . The control group maintained their normal physical activities throughout the experimental period, Moreover, all subjects were not allowed to reduce or raise their daily sport activities be. tween pretests and posttests.
SMT

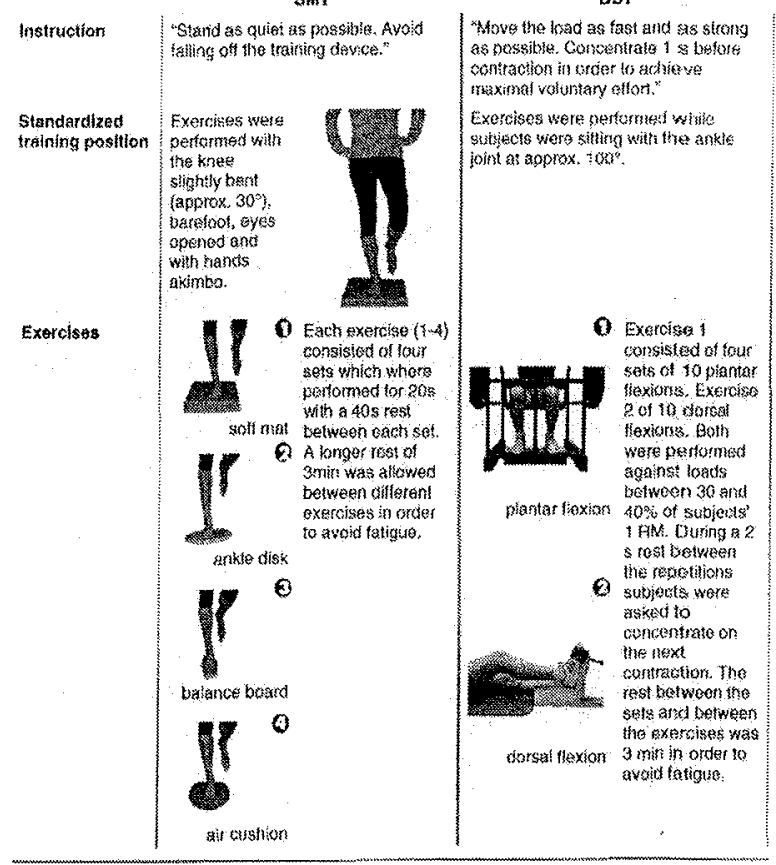

Ficuke 1. Training protocols for sensorimotor training (SMT) and ballistic strength training (BST). Exarcises were performed with the right leg except exercise 1 of BST, which was performed with both less simultaneousily. The total duration of each training session (SMT and BST) was 60 min. The warm-up and cool-down lasted for 10 min each on a bicyele dynamometer at $100 \mathrm{~W}$.

Measurement of MVC and Maximal RFD. Maximum voluntary isometric ankle plantar flexion strength was measured on an isokinetic system (Isomed 2000, D.\&R. Ferstl GmbH, Hemau, Germany). The maximum error of the torque sensor was $<0.2 \%$. The subjects were positioned on the seat of the isokinetic device with the hip and the knee angle adjusted to $90^{\circ}$ and the ankle angle to $100^{\circ}$. The waist, the thigh, and the shank were fixed on the isokinetic system and the subjects were asked to cross their arms in front of their chest. The exact position of each subject was documented and saved in the isokinetic system, so that it was identical in the pretraining and posttraining conditions. Testing was performed only on the right leg. A warm-up period of 10 minutes on: a bicycle ergometer at $100 \mathrm{~W}$ was mandatory for each sub. ject. After the ergometer warm-up, 3 to 5 submaximal isometric contractions were allowed for each subject to get accustomed to the testing procedure. Thereafter, each subject performed 5 plantar flexions. The two first trials had to be performed with submaximal effort. According to the visual inspections of the first two trials and of the resulting torque time curve, the subjects were instructed to perform a proper technical execution of the contraction. The last 3 trials of each set were performed with maximal voluntary effort. For each of these trials, subjects were thoroughly instructed to act "as forcefully and fast as possible,"

The torque signal and the EMG signals were sampled simultaneously at $1 \mathrm{kHz}$. The raw unfiltered signals were analog-to-digital converted (DAQ700, National Instru. ments, Austin, TX) and stored on a PC. During later off- 
line analysis, the torque signal was filtered by a digital fourth-order recursive Butterworth low-pass filter, upper cutoff frequency $50 \mathrm{~Hz}$.

Maximum voluntary isometric strength (MVC) and EMG were measured for each of the last 3 maximal isometric contractions and afterwards reported as mean values of the respective trials. Onset of torque was determined when torque exceeded $2 \mathrm{~N}$.m. Contractile impulses were defined as integrals of the torque-time curve from 0 to 50,50 to 100,100 to 150 , and 150 to $200 \mathrm{~ms}$ relative to the onset of force production (Figure 2A). Contractile impulse is of functional importance because it constitutes segmental angular velocity in nonisometric conditions. The significance of this parameter has been discussed in detail by Aagaard et al. (1). The RFDmax was defined as the maximal slope of the torque time curve (dT/dt) and time to reach $\mathrm{RFDmax}$ was determined relative to the onset of torque (Figure 2B).

Electromyography. Bipolar surface electrodes (Hellige, Freiburg, Germany; diameter $10 \mathrm{~mm}$, center-to-center distance $25 \mathrm{~mm}$ ) were placed over $\mathrm{M}$. soleus (SOL), M. gastrocnemius medialis (GAS), and TIB of the right leg. The longitudinal axes of the electrodes were in line with the presumed direction of the underlying muscle fibers. All electrode positions were carefully determined and marked to ensure identical pretraining and posttraining recording sites. Interelectrode resistance was kept below $5 \mathrm{k} \Omega$ by means of shaving, light abrasion, and degreasing and disinfecting the skin. The EMG electrodes were directly connected to custom-built differential preamplifiers (gain 200, input impedance 4,000 $\mathrm{M} \Omega$, common mode rejection $75 \mathrm{~dB}$ at $60 \mathrm{~Hz}$ ) and taped to the skin. The preamplified signals were transmitted via shielded cables to the main amplifier (band-pass filter [10 $\mathrm{Hz}$ to $1 \mathrm{kHz}$ ], gain 6.25 (resulting in an overall gain of 1,250$]$ ) and recorded with $1 \mathrm{kHz}$.

The onset of EMG integration was set $30 \mathrm{~ms}$ prior to the onset of torque to account for the occurrence of electromechanical delay. Absolute contractile EMG was determined as mean average voltage (MAV) in the distinct time intervals of -30 to 20,20 to 70,70 to 120 , and 120 to $170 \mathrm{~ms}$ relative to the onset of torque as well as 100 $\mathrm{ms}$ before and after MVC (MAV.MVC; Figure 2C). Normalized MAV values were calculated as MAV relative to MAV_MVC (expressed as percentage of MAV_MVC) for each trial. The frequency domain of the EMG signal of the first $200 \mathrm{~ms}$ relative to the onset of torque and 100 $\mathrm{ms}$ before and after MVC was analyzed by fast Fourier transformation (37). Only frequencies with a power exceeding $30 \%$ of peak power were used to determine the median frequency (MF).

Supramaximal nerve stimulation. The complete H-reflex recruitment curve was recorded in a sitting position with the ankle joint at $100^{\circ}$ before and after the training. The foot was strapped by a snowboard binding system to a foot plate dynamometer. H-reflexes were evoked by a 0.5 -ms current pulse applied to the tibial nerve in the popliteal fossa with a constant current square wave stimulator (Digitimer DS7; Digitimer, Hertfordshire, UK). A large $10 \times 5$-cm carbon rubber anode was placed below the patella and a small 2-cm-diameter electrode was used as a cathode. A suitable position for stimulation was located by carefully modifying cathode position in the popliteal fossa and simultaneously monitoring the SOL and the TIB EMG on an oscilloscope. Finally, the cathode was fixed, when a stable $\mathrm{H}$-reflex with minimal stimulus current and no TIB EMG activity was obtained. Electrode
A
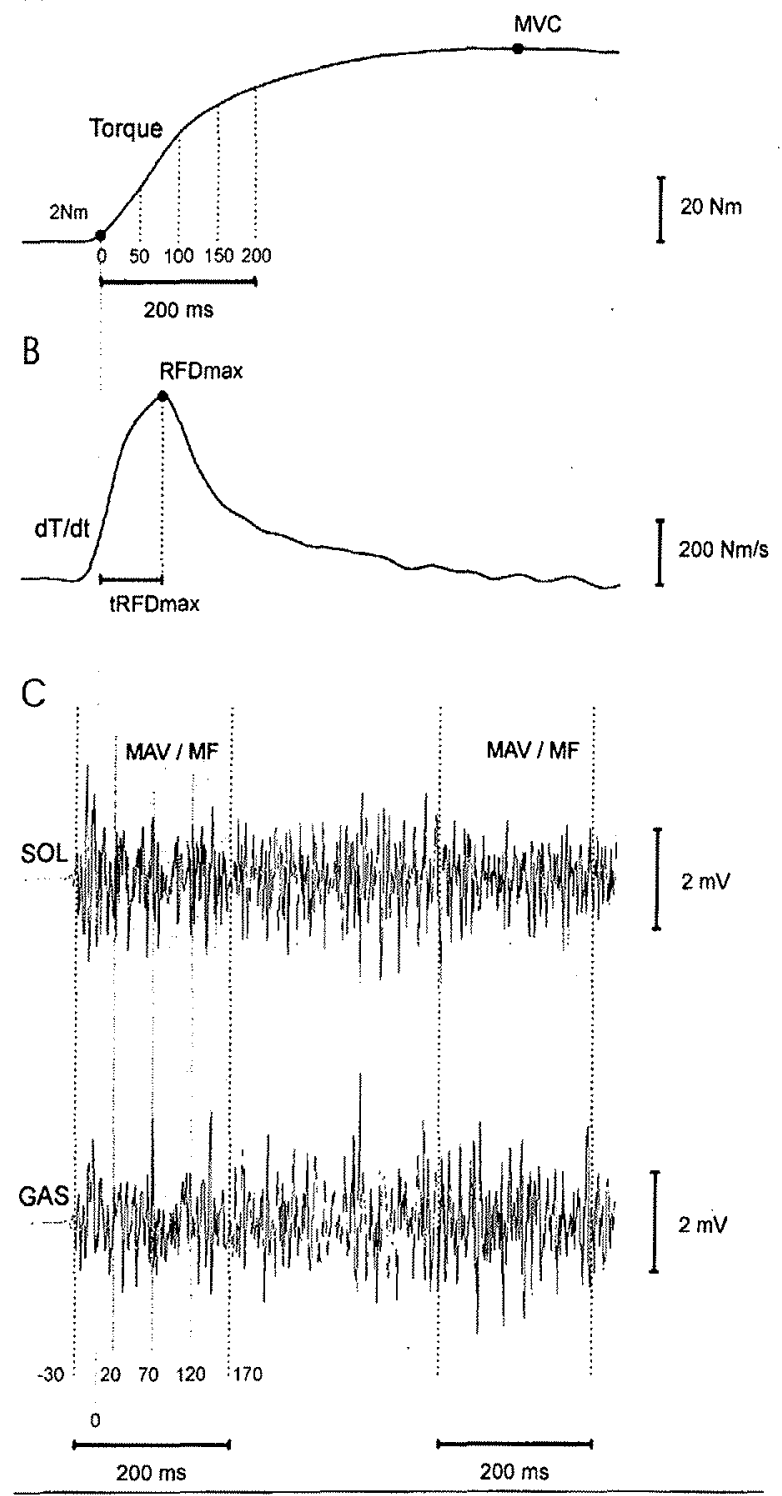

Figure 2. Torque and electromyography (EMG) signals of 1 subject recorded during a trial of maximal isometric plantar flexion in the isokinetic device. (A) Time 0 corresponds to the onset of the torque curve, which was determined when torque exceeded a value of $2 \mathrm{~N} \cdot \mathrm{m}$. The highest torque value indicated maximum voluntary isometric strength (MVC). Contractile impulses were defined as integrals of the torque-time curve from 0 to 50,50 to 100,100 to 150 , and 150 to $200 \mathrm{~ms}$. (B) Rate of force development (RFD) was derived as the slope of the torque-time curve ( $\mathrm{dT} / \mathrm{d} \mathrm{t})$. The highest value indicated RFDmax. The tRFDmax was calculated as the time interval from torque onset to RFDmax. (C) M. soleus (SOL) and M. gastrocnemius (GAS) EMG were analyzed in the time interval of $100 \mathrm{~ms}$ prior to $100 \mathrm{~ms}$ following MVC and from $30 \mathrm{~ms}$ prior to $170 \mathrm{~ms}$ following torque onset. The beginning of EMG analysis was set $30 \mathrm{~ms}$ prior to torque onset in order to account for electromechanical delay. For both time intervals, median frequencies of the power spectrum (MF) as well as mean amplitude voltage (MAV) were calculated. Moreover, the time interval from -30 to $170 \mathrm{~ms}$ was subdivided into $50-\mathrm{ms}$ intervals in order to gain detailed insight into the early phase of contraction. 
positioning was done in a standing position and checked in the sitting position before starting the experiment. During the experiment, the electric current was progressively increased until the maximal direct motor response (Mmax) was obtained. The Mmax was verified at least 1 time by increasing stimulation intensity beyond the intensity required for obtaining Mmax. The EMG signals of SOL, GAS, and TIB as well as the torque signal were recorded with $4 \mathrm{kHz}$. The mechanical response of supramaximal nerve stimulation was identified and the torque signal was filtered using a digital fourth-order recursive Butterworth low-pass filter, with a cutoff frequency of 50 $\mathrm{Hz}$. The onset of torque development was determined when the signal level exceeded $0.1 \mathrm{~N} \cdot \mathrm{m}$. From the maximal twitch response, peak torque (PT) and contraction time ( $\mathrm{Ct}$, defined as the time period from onset of torque to PT) were determined. Maximal rate of torque development (d[T]/d[t]max) was calculated as the maximal slope of the torque time curve (Figure 3 ).

\section{Statistical Analyses}

Data are presented as group mean values $\pm S E$. The data were analyzed by analysis of variance (ANOVA). The effect of the different interventions on muscle strength and neural activation were analyzed by means of ANOVA for repeated measures (3 [group] $\times 2$ [time] and 3 [group] $\times$ 2 [time] $\times 2$ [muscle], respectively). After an overall $F$ value was found to be significant $(p<0.05)$, preplanned a priori contrasts were performed to evaluate significant pretraining-posttraining changes in each group and differences in time between groups (Figure 4 and Tables 1 and 2). An ANOVA for repeated measures (3 [group] $\times 4$ [time interval]) was used to assess gains in contractile impulse and the corresponding EMG in distinct time in tervals at the beginning of contraction. In case of significance, Tukey post hoc tests were calculated to detect differences between groups (Figure 5). All analyses were ex. ecuted using SPSS 13.0 software (SPSS, Inc., Chicago, IL).

\section{RESULTS}

\section{Adaptations of Strength and Agonistic}

\section{Neuromuscular Activation}

The different training interventions caused various adaptations of the neuromuscular system. Figure 4 lists the most important parameters of torque and corresponding muscular activation before and after the training for the two training groups as well as for controls.

Whereas MVC and the corresponding agonistic neural activation (MAV and MF $100 \mathrm{~ms}$ prior to $100 \mathrm{~ms}$ following MVC) remained unchanged following BST and SMT (Fig. ure $4 \mathrm{D}-\mathrm{F}$ ), specific adaptations were found considering the beginning of contraction (Figure 4A-C). The RFDmax increased by $48 \pm 16 \%(p<0.01)$ in the BST group and by $14 \pm 5 \%(p<0.05)$ in the SMT group. Increased RFDmax was accompanied by decreased time to reach RFDmax (tRFDmax) for the BST group ( $14 \pm 5 \%$; from $58 \pm 3$ to $49 \pm 2 \mathrm{~ms} ; p<0.01)$ as well as for the SMT group (14 $\pm 3 \%$; from $55 \pm 4$ to $47 \pm 3 \mathrm{~ms} ; p<0.01$ ). Concerning tRFDmax, no differences were found between the training groups. Absolute agonistic EMG at the onset of activity $(-30$ to $70 \mathrm{~ms}$ after the onset of contraction) increased after BST. This increase was found for SOL ( 77 $\pm 15 \% ; p<0.05)$ as well as for GAS $(69 \pm 25 \% ; p<0.05)$. The MF of SOL and GAS increased after SMT. Gains were $13 \pm 4 \%(p<0.05)$ for SOL and $45 \pm 22 \%(p<0.05)$
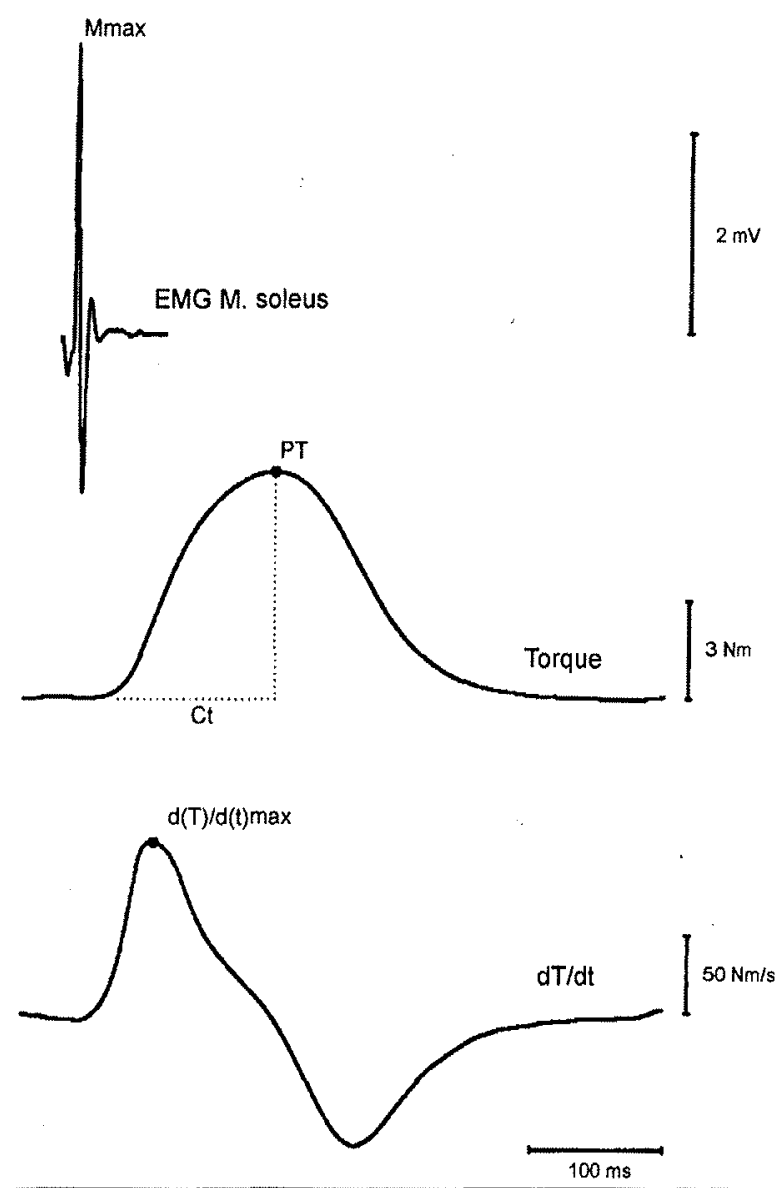

Figure 3. Torque and soleus electromyography (EMG) of 1 subject recorded following supramaximal stimulation of the tibial nerve. The Mmax was determined as the peak-to-peak amplitude of EMG response. Contraction time (Ct) was calculated as the time interval between onset of torque (when the signal level exceeded $0.1 \mathrm{~N} \cdot \mathrm{m}$ ) and peak torque (PT). Additionally, maximal rate of torque development (d[T] $d[t] \max )$ was determined at the maximal slope of the torquetime curve.

for GAS. After BST, MF increased only for GAS $(45 \pm$ $21 \% ; p<0.05$ ). Preplanned a priori contrasts revealed that gains in RFDmax and MAV at the beginning of contraction $(-30$ to $70 \mathrm{~ms})$ were statistically different between the training groups ( $p<0.05$; Table 1$)$.

To get a more detailed insight in training-specific adaptations concerning the first $200 \mathrm{~ms}$ of contraction, changes in the torque time course are depicted as mean group values in Figure 5. In 10 out of 11 subjects for the BST as well as for the SMT group, a distinct peak in torque increase was observed after the training when compared to pretraining values, whereas in the CON group no increases in torque were observed. Group mean and SE for the peaks in amplitude and timing that were found after SMT and BST are displayed in Figure 5A. After BST, the maximum increase in torque, $57 \pm 15 \%$, was observed $46 \pm 5 \mathrm{~ms}$ after the onset of torque development. Very similarly, after SMT the maximum increase in torque, $32 \pm 7 \%$, was observed after $45 \pm 8 \mathrm{~ms}$.

Contractile impulses increased for both training groups at the beginning of muscular contraction (Figure 


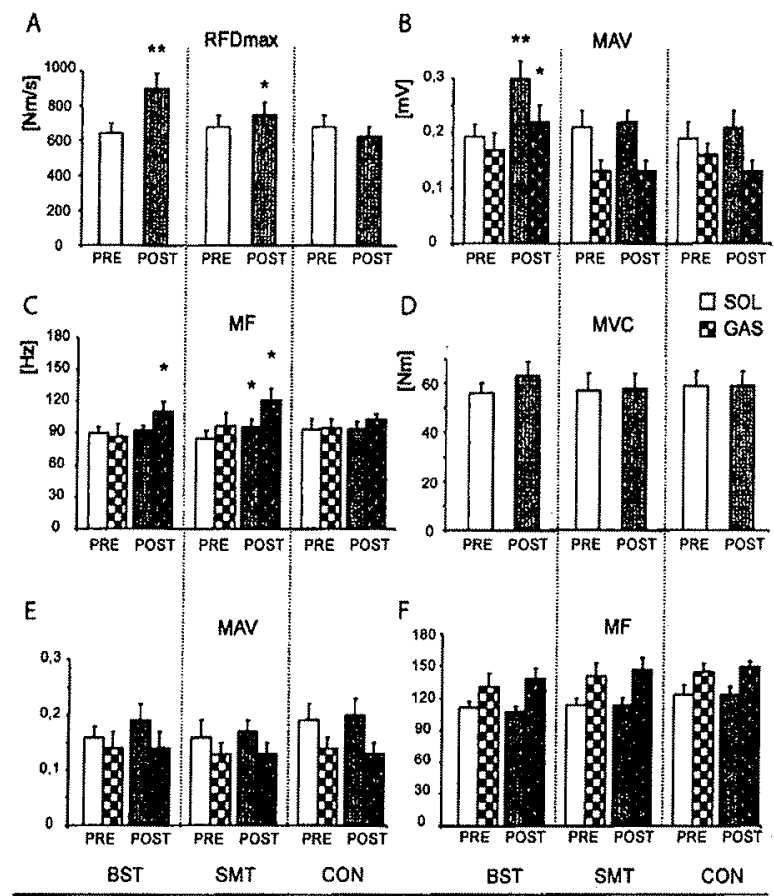

Figure 4. Most important parameters of the torque-time curve before and after 4 weeks of training as well as in controls. (A) Maximum rate of force development (RFDmax) increased following ballistic strength training (BST) and sensorimotor training (SMT), respectively. (B)

Electromyography (EMG) mean amplitude voltage (MAV) increased for both $\mathrm{M}$. soleus (SOL) and $\mathrm{M}$. gastrocnemius (GAS) muscles in the time interval -30 to $70 \mathrm{~ms}$ following BST only. (C) Median frequencies (MF) determined in the time interval -30 to $170 \mathrm{~ms}$ increased following SMT for SOL and GAS and following BST only for GAS. (D-F) Maximal voluntary contraction (MVC) as well as MAV and MF in the time interval $200 \mathrm{~ms}$ around MVC remained unchanged following BST and SMT, respectively. No changes were found for the control group. Pretraining and posttraining differences: $* p<0.05$ and $* * p<0.01$.

TABLE 1. Analysis of training and muscle-specific adaptation in explosive strength (Figure $4 \mathrm{~A}-\mathrm{C}$ ). Significant training effects (time) were demonstrated for maximum rate of force development (RFDmax) as well as for mean amplitude voltage (MAV) and median frequencies (MF) at the beginning of contraction ( -30 to $70 \mathrm{~ms}$ and -30 to $170 \mathrm{~ms}$, respectively). Moreover, changes over time were significantly different between the groups (time $\times$ group). Preplanned a priori contrasts revealed significant differences in adaptation between the sensorimotor training (SMT) and ballistic strength training (BST) groups for RFDmax and MAV. Concerning muscle-specific adaptation, a significant time $\times$ muscle effect occurred for MAV values only. However, muscle-specific changes in MAV were not caused by a certain training regimen, as indicated by no significant time $\times$ group $\times$ muscle effect. *

\begin{tabular}{lccc}
\hline \multicolumn{1}{c}{ ANOVA } & RFDmax & MAV & MF \\
\hline Time & $p<0.01$ & $p<0.05$ & $p<0.01$ \\
Time $\times$ group & $p<0.01$ & $p<0.01$ & $p<0.05$ \\
Time $\times$ muscle & & $p<0.05$ & ns \\
Time $\times$ group $\times$ muscle & & ns & ns \\
A priori contrast (SMT vs. BST) & $p<0.05$ & $p<0.05$ & ns \\
\hline
\end{tabular}

* ANOVA $=$ analysis of variance; $\mathrm{ns}=$ not significant.
TABLE 2. Contractile properties of the plantar flexors. The table displays peak twitch torque (PT), maximal rate of torque development ( $\mathrm{d}[\mathrm{T}] / \mathrm{d}[\mathrm{t}] \mathrm{max}$ ), contraction time (Ct), and the corresponding maximal direct motor response (Mmax) amplitudes for M. gastrocnemius medialis (GAS) and M. soleus (SOL).*

\begin{tabular}{|c|c|c|}
\hline \multirow[b]{2}{*}{ Variable } & \multicolumn{2}{|c|}{ Mean $\pm S E$} \\
\hline & Before training & After training \\
\hline \multicolumn{3}{|l|}{$\mathrm{PT}(\mathrm{N} \cdot \mathrm{m})$} \\
\hline BST & $7 \pm 1$ & $7 \pm 1$ \\
\hline SMT & $8 \pm 1$ & $8 \pm 1$ \\
\hline CON & $9 \pm 2$ & $9 \pm 2$ \\
\hline \multicolumn{3}{|c|}{$\mathrm{d}(\mathrm{T}) / \mathrm{d}(\mathrm{t}) \max \left(\mathrm{N} \cdot \mathrm{m} \cdot \mathrm{s}^{-1}\right)$} \\
\hline BST & $120 \pm 20$ & $120 \pm 20$ \\
\hline $\mathrm{SMT}$ & $180 \pm 30$ & $150 \pm 20$ \\
\hline CON & $160 \pm 40$ & $170 \pm 30$ \\
\hline \multicolumn{3}{|l|}{$\mathrm{Ct}(\mathrm{ms})$} \\
\hline $\mathrm{BST}$ & $112 \pm 5$ & $106 \pm 3$ \\
\hline SMT & $100 \pm 5$ & $103 \pm 4$ \\
\hline CON & $112 \pm 9$ & $110 \pm 8$ \\
\hline \multicolumn{3}{|l|}{$\operatorname{Mmax}(\mathrm{mV})$} \\
\hline \multicolumn{3}{|l|}{ SOL } \\
\hline $\mathrm{BST}$ & $5.5 \pm 0.7$ & $5.5 \pm 0.8$ \\
\hline SMT & $5.8 \pm 0.5$ & $5.4 \pm 0.4$ \\
\hline $\mathrm{CON}$ & $5.7 \pm 0.6$ & $6.1 \pm 0.7$ \\
\hline \multicolumn{3}{|l|}{ GAS } \\
\hline BST & $6.4 \pm 0.6$ & $6.6 \pm 1.0$ \\
\hline SMT & $6.3 \pm 0.4$ & $6.2 \pm 0.6$ \\
\hline $\mathrm{CON}$ & $5.5 \pm 0.9$ & $5.5 \pm 0.5$ \\
\hline
\end{tabular}

* BST $=$ ballistic strength training; SMT = sensorimotor training; $\mathrm{CON}=$ control.

5B). After BST, contractile impulses increased by $39 \pm$ $11 \%(p<0.01), 42 \pm 14 \%(p<0.05), 29 \pm 11 \%(p<0.05)$, and $23 \pm 10 \%(p<0.05)$ in the time intervals 0 to 50,50 to 100,100 to 150 , and 150 to $200 \mathrm{~ms}$. Respective increases after SMT were $22 \pm 6 \%(p<0.01), 19 \pm 5 \%(p<$ $0.05)$, and $13 \pm 5 \%(p<0.05)$ in the time intervals 0 to 50,50 to 100 , and 100 to $150 \mathrm{~ms}$ after onset of torque development. Gains in contractile impulse were accompanied by gains in normalized contractile EMG of SOL and GAS at the onset of contraction following BST but not after SMT (Figure 5C). The MAV of SOL increased only for the first $100 \mathrm{~ms}$ of contraction (in the time intervals -30 to 20 and 20 to $70 \mathrm{~ms})$ by $38 \pm 12 \%(p<0.01)$ after BST. Similar increases were found for GAS, with gains in MAV of $73 \pm 23 \%(p<0.05)$ for the respective time period (Figure 5D). Tukey's post hoc tests revealed a significant difference in MAV for both SOL and GAS between the training groups considering the time interval 20 to $70 \mathrm{~ms}(p<0.05)$. Following BST, SOL, and GAS, EMG was enhanced, whereas it remained unaffected following SMT.

\section{Adaptations of Antagonistic Neuromuscular Activation}

Normalized TIB EMG increased after BST for the following time intervals: -30 to $20 \mathrm{~ms}(68 \pm 17 \% ; p<0.01)$, 20 to $70 \mathrm{~ms}(37 \pm 15 \% ; p<0.01)$, and 70 to $120 \mathrm{~ms}(49$ $\pm 18 \% ; p<0.01)$. No changes were observed after SMT or for CON.

\section{Muscular Adaptations (Single Twitches)}

Contractile properties of SOL and GAS were examined before and after the training period using single electrical supramaximal nerve stimulations. Neither PT, Ct, nor 

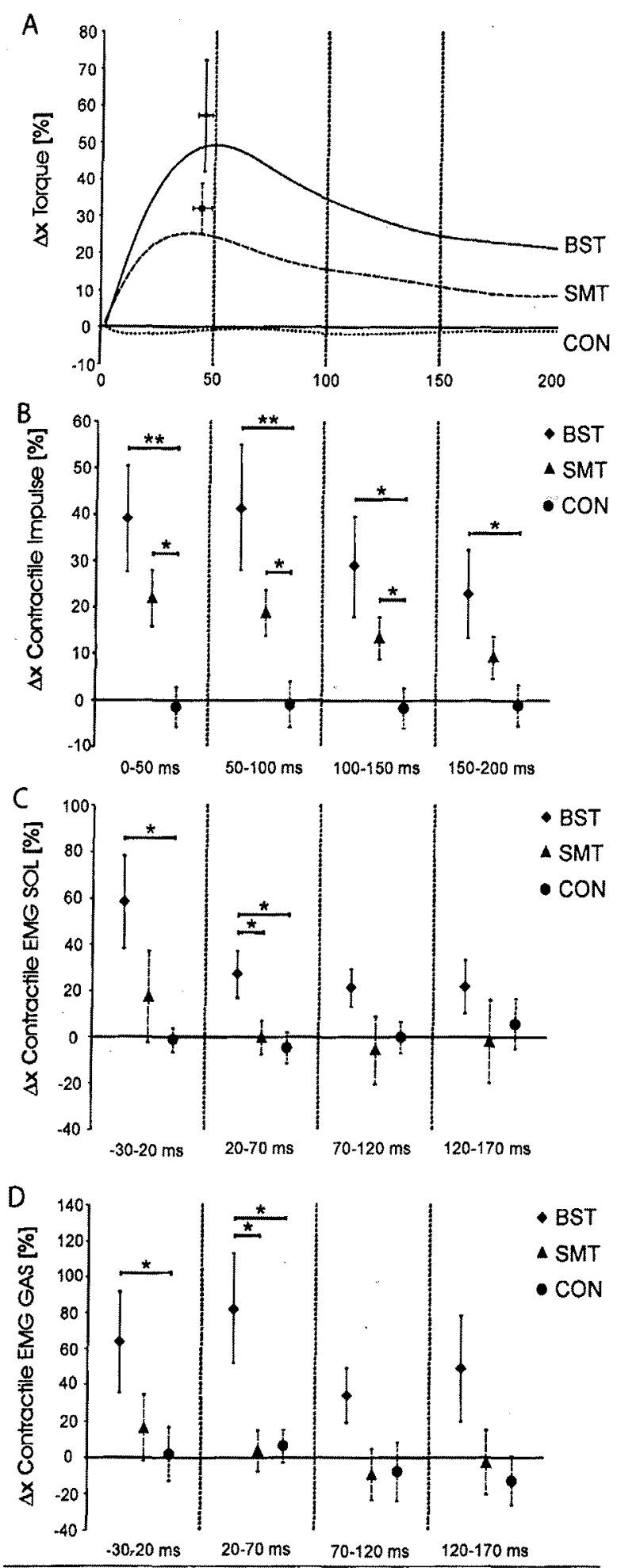

Fraure 5. (A) Changes (gain in \%) in torque as mean group values during the first $200 \mathrm{~ms}$ of torque production following 4 weeks of ballistic strength training (BST) or sensorimotor training (SMT) and in controls (CON). The highest gain in torque after BST and SMT was determined individually, and group means of those peaks in height and time of occurrence are displayed. (B) Gain in contractile impulse was determined
$\mathrm{d}(\mathrm{T}) / \mathrm{d}(\mathrm{t}) \max$ changed after training. Moreover, maximal M-wave peak-to-peak amplitudes of both muscles remained unchanged for the training groups and for the control group (Table 2).

\section{Discussion}

Both SMT and BST resulted in specific adaptations at the beginning of a voluntary isometric contraction. It was shown that 4-week BST as well as SMT resulted in an increased RFDmax without considerable adaptations in MVC. However, increases in RFDmax and the corresponding EMG were more pronounced following BST compared to SMT. The results will be discussed with respect to possible underlying neuromuscular mechanisms.

Maximum strength remained unchanged after both training regimens, whereas RFDmax increased considerably. These specific adaptations are most likely explained by adaptations in neural drive during the time course of voluntary contraction (Figure 4). Increases in RFDmax were found to be higher after BST compared to SMT (Figure 4A; Table 1). However, comparable adaptations where found for the contractile impulses determined at the beginning of contraction (Figure $5 \mathrm{~b}$ ). During single-joint movements, the contractile impulse is directly proportional to the angular velocity that would be reached in a nonisometric condition. Therefore, this parameter was suggested to be of functional importance to assess strength capacity at the onset of force production (1). An increase in contractile impulse of approximately $40 \%$ after BST and $20 \%$ after SMT during the onset of muscular activation $(-30$ to $70 \mathrm{~ms})$ can be interpreted as an increase in maximal unloaded movement speed at the same extent. This enhanced power capacity at the beginning of muscular contraction was clearly related to the training itself, as the control group did not show any changes.

Interestingly, improvement rates after SMT were lower compared to those reported in an earlier study in which subjects performed SMT with ski boots (17). In this specific study, after 4 weeks of training a $33 \%$ increase in RFDmax was observed for a voluntary isometric leg press exercise, which is much higher than the $14 \%$ increase in the present study. In our study, both training protocols were almost identical in duration and intensity. However, during a leg press exercise, the interaction and strength ability of various muscles determine overall strength. Adaptations on a single-muscle level might add up with enhanced intermuscular coordination and affect strength more clearly.

The findings after BST are in line with earlier studies that reported effects of this type of training on RFD (18, 36). Häkkinen and Komi (18) showed that after 24 weeks of BST the time to reach $30 \%$ of MVC during voluntary isometric knee extensions was reduced without any changes in MVC. Van Cutsem et al. (36) demonstrated an increased RFD after 12 weeks of dynamic training for vol-

$\leftarrow$

as the additional area covered by the torque-time curve after training compared with pretraining values in the time intervals of -30 to 20,20 to 70,70 to 120 , and 120 to $170 \mathrm{~ms}$ Gains in electromyography (EMG) of M. soleus (SOL) (C) and M. gastrocnemius (GAS) (D) were calculated as increases in normalized mean amplitude voltage (MAV) after training compared to pretraining values in the above-named time intervals. Group differences: ${ }^{*} p<0.05$ and $* * p<0.01$. 
untary isometric dorsiflexions but also an enhanced MVC. However, the training periods in these studies were much longer than 4 weeks and therefore it could not be excluded that changes in muscle properties contributed to adaptation in strength.

Duchateau and Hainaut (10) provided evidence for muscular adaptation following BST. The authors analyzed maximal evoked EMG (Mmax) together with the corresponding twitch response. They reported increased peak rates in tension development as well as reductions in contraction time after 3 months of moderate ballistic exercises in $M$. adductor pollicis. Interestingly, van Cutsem et al. (36) did not find any changes in the time course of the muscle twitch after the identical training period in TIB. Therefore, it was speculated that muscular adaptations after prolonged BST are muscle specific. In the present study, after 4 weeks of both BST and SMT, isometric twitches in response to a single supramaximal tibial nerve stimulation (Mmax) for triceps surae muscles showed no alterations in PT, $\mathrm{Ct}$, or time to reach PT. Moreover, Mmax of SOL and GAS remained unchanged after training. Therefore, increased RFD and contractile strength at the beginning of muscular contraction can be attributed basically to neural adaptive mechanisms.

Strength gains at the onset of plantar flexions were associated with enhanced SOL and GAS EMG (Figure 4B-C; Table 1). Although no differences were observed between the different training regimens considering MF, significant differences were detected in MAV (Table 1). This was reinforced by post hoc analysis of normalized MAV values in distinct time intervals at the beginning of contraction (Figure 5C-D). From 20 to $70 \mathrm{~ms}$ after the onset of contraction, which is exactly the time period to reach RFDmax, gains in MAV were significantly higher following BST compared with SMT. The results suggest that different mechanisms of adaptation are likely to be involved, which might account to some extent for the observed difference in RFDmax.

Neural mechanisms responsible for the increase in isometric strength could act within the muscle (intramuscular adaptation) or within muscle groups (intermuscular adaptation). In the present study, SOL and GAS EMG were found to be enhanced during the onset of contraction for a highly standardized isometric plantar flexion. This suggest that adaptations on a single-muscle level play a major role in a before/after training comparison. Single-MU recordings would be the only possibility to give direct evidence for specific adaptations on a motoneuron level. However, from other studies it is well known that the amplitude as well as the frequency information of the surface EMG encode control strategies like recruitment, discharge rates, and synchronization on a motoneuron level (12).

Evidence has been produced that MF is closely related to the average conduction velocity of active MUs $(2,6,24$, 34). Solomonow et al. (34) demonstrated that orderly stimulation of GAS MUs in the cat increased the MF of the EMG power spectrum linearly, whereas the increase in discharge rate of MUs did not affect MF. The authors suggested that recruitment control strategies that are responsible for an increase in contraction force could be assessed by determination of MF. Therefore, MF was used to quantify alterations in MU recruitment strategies after skill acquisition and between agonist and antagonist muscles in the dominant and in the nondominant arm respectively $(4,5)$.
Two results of the present study underline the abovementioned relation between MF and the recruitment of high-threshold MUs (Figure 4C,F). First, MF was lower during the onset of the contraction compared to MVC, which is well in line with the classic principle of Henneman et al. (19). Second, MF of SOL was lower compared to GAS, which could account for the higher proportion of high-threshold MUs in GAS compared to SOL (15). According to the fact that MF was enhanced following BST and SMT, it might be proposed that a shift in the excitation, i.e., an earlier recruitment of large MUs, potentially enhanced the RFD at a single-muscle level.

No differences in adaptation were found between the training regimens with respect to MF, whereas MAV was specifically increased only following BST (Table 1). Additional recruitment of previously silent MUs as well as increased discharge rates of already recruited MUs could explain an increased MAV after BST. Furthermore, higher activation amplitudes could result from improved lev. els of synchrony between single MUs. This was recently predicted by a computer model of muscle contraction (38). More functionally, it was assumed that MU synchrony plays an important role in increasing RFD on the basis of experiments on the so-called premovement silent period $(9,27)$. It was shown that a strong depression of EMG activity that occurred 50 to $60 \mathrm{~ms}$ before the beginning of a ballistic movement was associated with an increased RFDmax (27). On a motoneuron level, tonically active MUs are silenced during this period in order to ascertain a subsequent phasic innervation (9). In the present study, MAV values of SOL and GAS at the beginning of contraction were even higher compared to MAV values at MVC. Normalized MAV values increased from $120 \pm 10 \%$ before to $160 \pm 20 \%$ after the training in SOL. For GAS, similar ratios were observed (120 \pm 20 vs. $180 \pm 40$ ). Therefore, higher MAV at the beginning of contraction compared to MAV during MVC might indicate an increased synchronization of MUs at the beginning of contraction, because it seems unlikely that recruitment or discharge rates should be lower during MVC compared to the beginning of contraction. Although we were not able to demonstrate alterations on a motoneuron level, it seems quite reasonable to assume that enhanced synchronization between MUs might have contributed to increased RFD.

Interestingly, adaptations in MAV were found to be muscle specific for SOL and GAS (Table 1). However, as no significant group $\times$ time $\times$ muscle effect was detected, muscle-specific adaptation was not connected to a certain training regimen. This indicates that muscle specificity might instead be attributed to differences in functional properties of the muscles (15). Previously it was shown that GAS is preferentially activated during fast movements (11) and during the eccentric phase of a movement (28). Moreover, there is some evidence from experiments with rhesus monkeys that both muscles adapt specifically to microgravity conditions in their relative recruitment (31). Therefore it is suggested that altered neural control of the synergistic muscles involved in plantar flexion can occur on the basis of their functional prerequisites.

Specific adaptations following BST were not limited to synergistic muscles but also were prominent for the antagonist. The MAV values of TIB increased at the beginning of contraction. From a functional point of view, it has been argued that an increased agonist/antagonist coactivation offers specific advantages. During ballistic movements, an increase in antagonistic EMG has been 
reported together with an increase in velocity and loading $(7,8)$. Thus, this increased coactivation could enhance contractile performance (7) or just help to stiffen and protect the joint (35).

The SMT was characterized by a complex activation pattern in the muscles encompassing the ankle joint in order to maintain or regain balance. Therefore, it was not possible to quantify exactly the amount of training load and volume performed by the respective subjects during the training period. It could be estimated that during SMT work should be lower compared with BST because during SMT only minor joint movements take place, whereas during BST magnitude of movement is much higher. However, durations of muscular loading and muscular recovery were identical in the protocols. From a more functional point of view, it was important to ascertain the intensity of both training regimens to a maximal level. This was achieved by 4 training sessions per week over the time period of 4 weeks, which should result in maximal adaptations for both training regimens.

A fundamental difference between SMT and BST may basically be seen in the amount of afferent contributions during the training itself. Although BST was performed sitting on a chair and the exercises comprised volitional activations of the muscles, SMT required an upright stance with the muscles being activated in order to control balance on instable devices. Therefore, BST was characterized by almost no afferent input, whereas during SMT afferent contributions occurred frequently and were supposed to play an important role for an adequate activation of the stabilizing muscles. New findings emphasize that recruitment thresholds as well as discharge rates of motoneurons depend on the amount of afferent input (16). By analyzing single-MU recordings, Grande and Cafarelli (16) were able to show that both recruitment thresholds and discharge rates were significantly lower during reflex contractions compared to voluntary contractions at the same force level. Moreover, additional Ia afferent input, provided to the motoneuron pool during voluntarily contracting the muscle, resulted in reduced discharge rates of active MUs while concomitantly recruiting others. The functional significance of spinal excitatory contributions to assist the voluntary descending drive in order to produce force as fast as possible was pointed out by Meunier and Pierrot-Deseilligny (25). The authors showed that presynaptic inhibition of la afferents is modulated in line with the steepness of RFD during voluntary ramp and hold contractions. During a ramp contraction of $50 \%$ MVC the supplementary heteronymous Ia facilitation was approximately 4 times higher compared to a ramp contraction of $12 \%$ MVC.

In contrast to these findings, it was suggested recently that primarily supraspinal centers are involved in the planning and execution of muscle contraction during BST (21). Moreover, Behm and Sale (3) showed that the intended rather than the actual movement velocities were responsible for enhanced explosive muscle strength, and Yue and Cole (39) reported similar strength increases after 4 weeks of isometric compared to mental training. These results suggest that after BST important underlying neural adaptations result from practice effects on central motor planning. Interestingly, not only discharge rates and recruitment depend on the ratio of spinal and corticospinal contributions (16). Recently, evidence for the contribution of the corticospinal pathway to MU synchronization was provided by two studies that compared $\mathrm{MU}$ synchronization between patients with conditions that af- fect the corticospinal pathway and controls $(13,33)$. Therefore, the difference in afferent input during both exercise types might have resulted in different recruitment of MUs and therefore caused different neural adaptations on a single muscle level.

In conclusion, the present study demonstrated increased RFDmax after 4-week BST and SMT during maximal isometric plantar flexions. Increased RFDmax was basically related to neural adaptations, as was shown by unchanged effects of supramaximal nerve stimulations. Following BST, training-induced increases in RFDmax were accompanied by increases in MF and MAV at the beginning of muscle contraction, whereas following SMT increases were observed only in MF. This clearly indicates differences in neural mechanisms of adaptation between the two training regimens that are most probably caused by differences in afferent input during the training.

\section{Practical Applications}

The present study basically extends our knowledge of explosive muscle strength adaptability. Significant increases in RFD and contractile impulses were observed after only 4 weeks of balance training (SMT). This impressively contradicts the paradigm that generally has been.constituted for classical weight training: Adaptations in explosive muscle strength require voluntary activation of the respective muscles with the intention to accelerate a load as fast as possible $(3,39)$. Interestingly, EMG data suggest that BST and SMT involve different mechanisms of neural adaptation. This would offer the possibility of combining both training regimens with the intention to further increase the gain in explosive muscle strength. Although the subjects in our study were not at the elite level, similar adaptations could be assumed for highly trained athletes (29). It is suggested that SMT could be used complementarily to BST in sports that require high demands in explosive muscle power. Moreover, for athletes who need contractile explosive properties together with precise movement control, this type of combination might be an extra benefit. A striking example is ball sports in which jumps have to be performed coexistent with the attempt to adjust the direction correctly with respect to different players and ball positions. It could be supposed that SMT would lead to an adequate integration of various proprioceptive signals in order to adjust the motor program to the demands required in distinct situations.

Beside the application of SMT supplemental to BST for athletes, SMT seems to be of major importance in lesstrained subjects (e.g., in the elderly). It is well known that the capacity for explosive force production, especially of the leg and foot extensor muscles, declines drastically with increasing age (23). Recently, it has been shown that this decreased ability of RFD is accompanied by impaired balance control (20). On the basis of their results, Izquierdo et al. (20) recommended strength training in combination with explosive exercises as well as specific balance exercises in order to reduce the risk of falling. On the basis of our results, SMT seems to be highly adequate as a countermeasure of these age-related compromises. Moreover, because of the nature of the training regimen itself, increases in RFD could be achieved with only minor strains of the muscle. This might be of importance, especially for less exercise-experienced people. 


\section{REFERENCES}

1. AagaARd, P. E.B. STMONSEn, J.L. ANdersen, P. MAGnusson, and P. DYHRE-POULSEN. Increased rate of force development and neural drive of human skeletal muscle following resistance training. J. Appl. Physiol. 93:1318-1326. 2002

2. ARENIYr-NiblSEN, L., ANd K.R. Milis. The relationship between mean power frequency of the EMG spectrum and muscle fibre conduction velocity, Electroencephalogr. Clin. Neurophysiol 60:130-134. 1985.

3. BEHM, D.G., AND D.G. SALE. Intended rather than actual movement velocity determines velocity-specific training response. J. Appl. Physiol. 74 359-368. 1993.

4. Bernardi, M., F. Felici, M. MARchetti, F. Montellantco, M.F. PI ACENTINI, AND M. SOLOMONOW. Force generation performance and motor unit recruitment strategy in muscles of contralateral limbs. J. Electromyogr. Kinesiol. 9:121-130. 1999.

5. BERnARdi, M., M. SOLOMONOW, G. NGUYen, A. SMith, AND R. BARATTA Motor unit recruitment strategy changes with skill acquisition. Eur. $J$ Appl. Physiol. Occup. Physiol. 74:52-59. 1996.

6. Broman, H., G. BrLotro, AND C.J. DE LUCA. Myoelectric signal conduction velocity and spectral parameters: Influence of force and time. $J$. Appl. Physiol. 58:1428-1437. 1985.

7. Carpentier, A., J. Duchateau, and K. Hainaut. Velocity-dependent muscle strategy during plantarflexion in humans. J. Electromyogr. Kinesiol. 6:225-233. 1996.

8. Carpentier, A., J. DuchateaU, and K. Hannaut. Load-dependent muscle strategy during plantarflexion in humans. J. Electromyogr. Kinesiol. 9:1-11. 1999

9. Conrad, B., R. Benecke, and M. Goemmann. Premovement silent period in fast movement initiation. Exp. Brain Res. 51:310-313.1983.

10. Duchatead, J., and K. Hainautr. Isometric or dynamic training: Differential effects on mechanical properties of a human muscle. J. Appl. Phys iol. 56:296-301. 1984 .

11. Duchateau, J S. Le Bozec, AND K. Hainaut. Contributions of slow and fast muscles of triceps surae to a cyclic movement. Eur. J. Appl. Physiol. Occup. Physiol. 55:476-481. 1986

12. Fanina, D., R. MERLETt, AND R.M. ENOKA. The extraction of neural strategies from the surface EMG. J. Appl. Physiol. 96:1486-1495. 2004.

13. Farmer, S.F., D.A. INGRAM, ANd J.A. STEPHENS, Mirror movements studied in a patient with Klippel-Feil syndrome. d. Physiol. 428:467-484. 1990 .

14. GoLthofER, A. Proprioceptive training: Considerations for strength and power production. In: Strength and Power in Sport. P.V. Komi, ed. Oxford, UK: Blackwell, 2003. pp. 331-342.

15. Golinick, P.D., B. SJodin, J. Karlsson, E. Jansson, and B. Saltin. Human soleus muscle: A comparison of fiber composition and enzyme activities with other leg muscles. Pfiugers Arch. 348:247-255. 1974.

16. Grande, G., AND E. CaFarelli. Ia afferent input altery the recruitment thresholds and firing rates of single human motor units. Exp. Brain Res. 150:449-457. 2003

17. Gruber, M., AND A. Gollhofer. Impact of sensorimotor training on the rate of force development and neural activation. Eur. J. Appl. Physiol. 92:98-105. 2004 .

18. HAKKHINEN, KK, AND P.V. KoMl. Training-induced changes in neuromuscular performance under voluntary and reflex conditions. Eur. J. Appl Physiol. Occup. Physiol. 55:147-155. 1986.

19. Henneman, E, G. Somdem, and D.O. Carpenter. Functional significance of cell size in spinal motoneurons. J. Neurophysiol. 28:560-580. 1965.

20. IZQUIERdo, M., X. AGUADO, R. GONZALEZ, J.L. LOPEZ, AND K. HAKkgNEN Maximal and explosive force production capacity and balance performance in men of different ages. Eur. J. Appl. Physiol. Occup. Phystol. 79: 260-267. 1999
21. Johannsen, P., L.O. Christensen, T. Sinkjaer, and J.B. Nielsen. Ce rebral functional anatomy of voluntary contractions of ankle muscles in man. J. Physiol. 535:397-406. 2001

22. KaneKo, M. T. Fuchimoto, $\mathrm{H}$. TOJ, and K. SuEt. Training effect of different loads on the force-velocity relationship and mechanical power output in human muscle. Scand. J. Sports Sci. 5:50-55, 1983.

23. Macaluso, A., AND G. DE Vtro. Muscle strength, power and adaptation to resistance training in older people. Eur. J. Appl. Physiol. 91:450-472 2004.

24. MrRtettri, R., M. KNafujt, AND C.J. DE LuCA. Myoelectric manifestations of fatigue in voluntary and electrically elicited contractions. $J$. Appl Physiol. 69:1810-1820. 1990.

25. Meuniek, S., AND E. PIERROT-DESEILlignY. Gating of the afferent volle of the monosynaptic stretch reflex during movement in man. J. Physiol 419:753-763. 1989 .

26. MortTANI, T., AND H.A. Devries. Neural factors versus hypertrophy in the time course of muscle strength gain. Am. J. Phys. Med. 58:115-130. 1979.

27. Moritani, T., and M. Shibata. Premovement electromyographic silent period and lalpha]-motoneuron excitability, J. Electromyogr. Kinesiol. 4 27-36. 1994

28. Nardone, A., C. Romano, AND M. Schieppat. Selective recruitment of high-threshold human motor units during voluntary isotonic lengthenin of active muscles. J. Physiol. 409:451-471. 1989.

29. NEwTON, R.U., W.J. KRAEMER, AND K. HAKKINEN, Effects of ballistic training on preseason preparation of elite volleyball players. Med. Sci Sports Exerc. 31:323-330. 1999.

30. Ross, A., M. LEVERITT, AND S. RIEK. Neural influences on sprint running. Training adaptations and acute responses. Sports Med. 31:409-125. 2001.

31. ROY, R.R., J.A. HODGSON, J. ARAgON, M.K. DAY, I. KOZLOVSKaYA, AND V.R. EDGFRTON, Recruitment of the Rhesus soleus and medial gastrocnemius before, during and after spaceftight. d. Gravit. Physiol. 3:11-15. 1996.

32. SCHMidTBLEICHER, D., AND G. HaRALAMBIE. Changes in contractile prop erties of muscle after strength training in man. Eur. J. Appl. Physiol. Occup. Physiol. 46:221-228. 1981.

33. SCHMIED, A., J. POUGET, AND J.P. VedeL. Electromechanical coupling and synchronous firing of single wrist extensor motor units in sporadic amyotrophic lateral sclerosis. Clin. Neurophysiol. 110:960-974. 1999.

34. Solomonow, M., C. Baten, J. Smit, R. Baratta, H. Hermens, R D'AMBrosia, AND H. ShoJ. Electromyogram power spectra frequencies associated with motor unit recruitment strategies. J. Appl. Physiol. 68 $1177-1185.1990$

35. TYLER, A.E., AND R.S. HUTTON. Was Sherrington right about co-contractions? Brain Res. 370:171-175. 1986.

36. Van Cutsem, M., J. Duchateau, and K. Hainaut. Changes in single motor unit behaviour contribute to the increase in contraction speed af ter dynamic training in humans. J. Physiol. 513:295-305. 1998.

37. VON TsCHARNER, V. Time-frequency and principal-component methods for the analysis of EMGs recorded during a mildly fatiguing exercise on a cycle ergometer. J. Electromyogr. Kinesiol. 12:479-492. 2002

38. YAO, W. R.J. FUGLEVAND, AND R.M. ENOKA. Motor-unit synchronization increases EMG amplitude and decreases force steadiness of simulated contractions. J. Neurophysiol. 83:441-452. 2000.

39. YUE, G., AND K.J. CoLE. Strength increases from the motor program Comparison of training with maximal voluntary and imagined muscle contractions. J. Neurophysiol. 67:1114-1123. 1992.

\section{Acknowledgment}

This project was supported in part by DFG grant SCHU1487/ 11.

Address correspondence to Markus Gruber, markus. gruber@sport.uni-freiburg.de. 